\title{
Sciatic Nerve Entrapment (Deep Gluteal Syndrome) as a Cause of Failed Back Surgery Syndrome: A Case Report
}

\author{
Yunoh Hwang ${ }^{1}$, Byung-chul Son ${ }^{1,2}$ \\ ${ }^{1}$ Department of Neurosurgery, Seoul St. Mary's Hospital, College of Medicine, The Catholic University of Korea, Seoul, \\ ${ }^{2}$ Catholic Neuroscience Institute, College of Medicine, The Catholic University of Korea, Seoul, Republic of Korea
}

Corresponding author: Byung-chul Son Department of Neurosurgery, Seoul St. Mary's Hospital, Catholic Neuroscience Institute, College of Medicine, The Catholic University of Korea, 222 Banpo-daero, Seocho-gu, Seoul 06591 Republic of Korea

Tel: +82-2-2258-6122

Fax: +82-2-594-4248

E-mail: sbc@catholic.ac.kr

Received: August 15, 2020

Revised: September 6, 2020

Accepted: September 17, 2020
Sciatic nerve entrapment, which is a cause of non-discogenic extraspinal sciatica is characterized by buttock pain and sitting intolerance. If significant asymptomatic lumbar stenosis in the lower lumbar spine is found in patients with buttock pain and sciatica, caused by sciatic nerve entrapment, it is possible that needless spinal surgery may have been recommended. A 72-year-old male presented with a 2-year history of severe buttock and sole pain in his right lower extremity. His pain did not improve after decompression and fusion surgery for severe lumbar stenosis. A pelvic magnetic resonance imaging (MRI) used to evaluate the failed back surgery syndrome revealed a type II variation between the sciatic nerve and piriformis muscle. Transgluteal decompression of the sciatic nerve completely eliminated chronic disability associated with right buttock and sole pain. The patient's pain was improved by sciatic nerve decompression through a transgluteal approach. Although vascular claudication, hip joint pathology, and peripheral neuropathy have been suggested in the differential diagnosis of lumbar stenosis, sciatic nerve entrapment involving the gluteal region should be suspected in patients presenting with buttock pain associated with sciatica, combined with severe lumbar stenosis based on MRI.

Key Words: Failed back surgery syndrome; Piriformis muscle syndrome; Sciatic nerve; Spinal stenosis

\section{INTRODUCTION}

Entrapment of the sciatic nerve in the gluteal region has long been known by the elusive name of "piriformis syndrome". Piriformis syndrome can be defined simply as a non-discogenic cause of sciatica due to sciatic nerve impingement through or around the piriformis muscle ${ }^{4)}$. Due to multiple etiologies underlying sciatic nerve entrapment in addition to its compression via the piriformis muscle, such as fibrous bands containing blood vessels, gluteal, and hamstring muscles that have no direct association with the piriformis muscle, the name "deep gluteal syndrome" has been suggested recently, ${ }^{9,15)}$. Deep gluteal syndrome is an underdiagnosed entity characterized by pain and/or dysesthesia in the buttock area, hip, or posterior thigh and/or radicular pain due to non-discogenic sciatic nerve entrapment in the subgluteal space ${ }^{9)}$. Therefore, piriformis syndrome can be regarded as a subtype of deep gluteal syndrome, implying that not all deep gluteal syndromes represent piriformis syndrome ${ }^{9)}$.

Not all patients presenting with sciatica are due to compre- ssion of the lumbar nerve root. Although sciatica was primarily attributed to sciatic nerve entrapment in the pelvis prior to $1934^{9,17,25)}$, a well-known report by Mixter and Barr ${ }^{16)}$ changed the focus of sciatica to lumbar nerve root compression by the herniated disc. Sciatica may be caused by factors unrelated to disc lesions such as piriformis syndrome and distal foraminal impingement ${ }^{6}$. Nevertheless, most patients with sciatica are only evaluated for spinal lesions using X-ray, computed tomography (CT), and magnetic resonance imaging (MRI) in a busy clinical setting. Conventional MRI of the lumbar segment of the spine is well established and provides an excellent imaging modality for patients with back and leg pain ${ }^{17)}$. However, if only lumbar spine MRI is performed without detailed medical history and physical examinations of sciatica patients, it increases the risk of unnecessary surgery when asymptomatic lumbar stenosis is detected. Severe radiological lumbar stenosis elevates the risk of failed back surgery syndrome (FBSS). We report a case of late diagnosis of sciatic nerve entrapment in a sciatica patient who underwent spinal fusion surgery due to coincident, severe radiological stenosis in the lumbar spine MRI. 


\section{CASE REPORT}

A 72-year-old man presented with a 2-year history of severe pain involving the right hip and sole. Dysesthetic pain occurred insidiously in the middle metatarsal area for no specific reason, which radiated to the heel area (Fig. 1A). The pain was described as tightening and tearing, and persisted throughout the day. His pain was more severe at night, especially when he was asleep. The patient experienced tearing pain involving his right sole, which disrupted his sleep at night for the last 2 years. He was treated for plantar fasciitis via orthopedic surgery, but the pain in the sole of the feet did not respond to medication or physical therapy. MRI of the right foot revealed no abnormalities.

One year after the occurrence of pain, the pain in the right hip and the sole became more severe, which greatly interfered with the activities of daily life and work. He was diagnosed with lumbar spinal stenosis at a spine clinic and was treated with regular medications and epidural blocks, without any improvement. He visited the neurosurgical spine clinic at a tertiary hospital and was treated with medications and S1 root block. The root block of the right S1 was not effective. Electromyography (EMG) revealed a radiculopathy of the right S1 nerve root. Severe stenosis of the L3/4 and L4/5 lumbar spine was again confirmed by MRI (Fig. 1B, C). A lumbar decompression and fusion surgery was performed due to medically refractory chronic pain in the right hip and sole, which started 2 years ago. Decompressive hemilaminectomies of right $\mathrm{L} 3 / 4$, L4/5, and L5/S1 and oblique lumbar interbody fusion at L3/4 were performed without any surgical complications or adverse effects (Fig. 1D). However, the pain in his right hip and sole continued without improvement. A myelographic CT scan of the lumbar spine performed postoperatively after 3 months did not show any residual stenosis. He was referred to the present author for unremitting pain.

In an interview, the patient complained of constant pressure and tearing pain on the right sole for the past 2 years, and additional pain in his lower right buttock started a year ago. The pain was constant while walking or resting and was very severe when sitting or driving for more than $30 \mathrm{~min}$. He explained that his occupation entailed a lot of work to driving, and his symptoms interfered with his job. When the pain intensified, tingling occurred on the side of the right calf. Physical examination revealed no motor weakness, hypesthesia, or allodynia. The patient's deep tendon reflex was normal with no limitation in straight leg raising test. Mild tenderness was observed in the sore spot on his right buttock. MRI of the pelvis was requested due to the possibility of sciatic nerve entrapment, considering the worsening of symptoms at night, severe pain upon sitting, and tenderness in the buttock.

MRI of the pelvis revealed a type 2 variation of the sciatic nerve according to the Beaton and Anson classification (Fig. $2 \mathrm{~A}, \mathrm{~B})$, at the peroneal division of the right sciatic nerve between the piriformis muscles (Fig. 2C). A decompression of the right sciatic nerve was planned due to significant entrapment of the sciatic nerve. The patient was placed prone position under the general anesthesia. The presumed course of the right sciatic nerve, according to the MRI images, was marked in the buttock under fluoroscopic vision. Subcutaneous fat, gluteal fascia, and the gluteus maximus muscle were dissected and retracted following an approximately $10 \mathrm{~cm}$, lazy S-shaped incision. The underlying piriformis muscle and the sciatic nerve were identified after dissection and cutting of the branches of the inferior gluteal artery and vein (Fig. 3A). The peroneal and tibial divisions of the sciatic nerve were confirmed with intraoperative nerve stimulation ( $2 \mathrm{~Hz}, 1-2 \mathrm{~mA}$ ). The lower part of the piriformis muscle was resected to reveal a nerve in the lateral side of the tibial division of the sciatic nerve (Fig. 3B). It was the peroneal division of the sciatic nerve, which was determined by the evoked EMG response on the tibialis anterior muscle via a monopolar stimulation $(2 \mathrm{~Hz}, 1-2 \mathrm{~mA})$. The remaining lower part of the piriformis muscle sandwiched between the peroneal division and the tibial division of the sciatic nerve was removed (Fig. 3B).

A subsequent dissection was performed proximal to the lateral margin of the sacrotuberous ligament, and the proximal
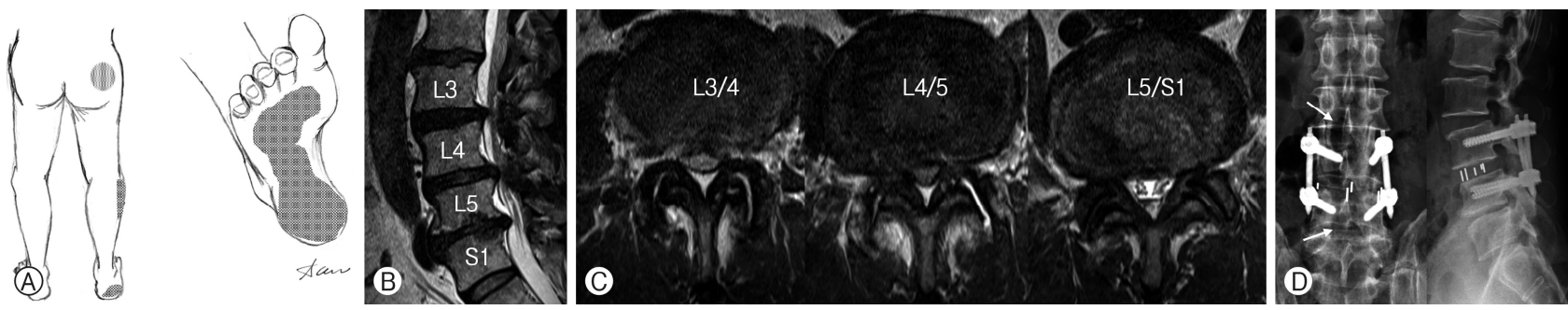

Fig. 1. Presentation of the patient with right buttock and sole pain. (A) Distribution of right leg pain. Black areas indicate pain location. (B) Sagittal T2-weighted magnetic resonance imaging (MRI) image of the lumbar spine revealed multi-level stenosis in the lumbar spine. Note significant spinal stenosis and disc protrusions in the lower lumbar spine. (C) Axial T2-weighted MRI image of the lumbar spine revealed significant narrowing of the spinal canal at L3/4, L4/5, and L5/S1 levels with degenerative disc protrusions. (D) Anterior-posterior (left) and lateral (right) $X$-rays showing an instrumented interbody fusion at $L 4 / 5$ and partial hemilaminotomies at $L 3 / 4$ and $L 5 / S 1$ (white arrows). 
piriformis muscle overlying the sciatic nerve was excised to the point where the sciatic nerve originated from the greater sciatic foramen (Fig. 3C). The overlying wound was closed in layers after confirming complete decompression of the tibial and peroneal divisions of the sciatic nerve with resection of the surrounding pyriformis and its tendinous structure.

The postoperative course was uneventful. During the postoperative hospital stay, the patient reported that the pain that had prevented him from falling asleep at night was gone, and he was able to sleep well. The severe pain in the sole also decreased by more than half from the day after the surgery. No weakness or sensory impairment was observed on his right leg and foot. The patient was discharged on day 4 after surgery, without any complications, except for minor discomfort in the operating area. The tearing pain in the soles of the right foot was reduced by about $80 \%$ after one month after surgery, no pain was found in the operation site, so there was no difficulty with sitting. No pain was experienced in the foot and buttock at 3 months after surgery, and no pain in the right hip occurred despite prolonged driving.

\section{DISCUSSION}

\section{Entrapment of the Sciatic Nerve and Sciatica}

Entrapment of the sciatic nerve at any point along its course results in a clinical syndrome of buttocks and leg pain known as sciatica, with a reported incidence as high as $43 \%{ }^{13,22,23)}$. It predominantly affects individuals in the 4th and 5th decades of life ${ }^{22,23)}$. The most common cause of sciatica is degenerative disc disease and osteoarthritic changes resulting in stenosis of the spinal canal and neural foramen ${ }^{23)}$. A less common and under-diagnosed cause of sciatica is sciatic nerve entrapment in the gluteal region (formerly called pyriformis syndrome) ) $^{3,520)}$. Classically, piriformis syndrome was thought to result from hypertrophy and inflammation of the pyriformis muscle involving an estimated $6 \%$ to $8 \%$ of all sciatica cases ${ }^{23)}$. The anatomical variations of the piriformis muscle are the most important among multiple causes of the sciatic nerve entrapment involving the subgluteal space.
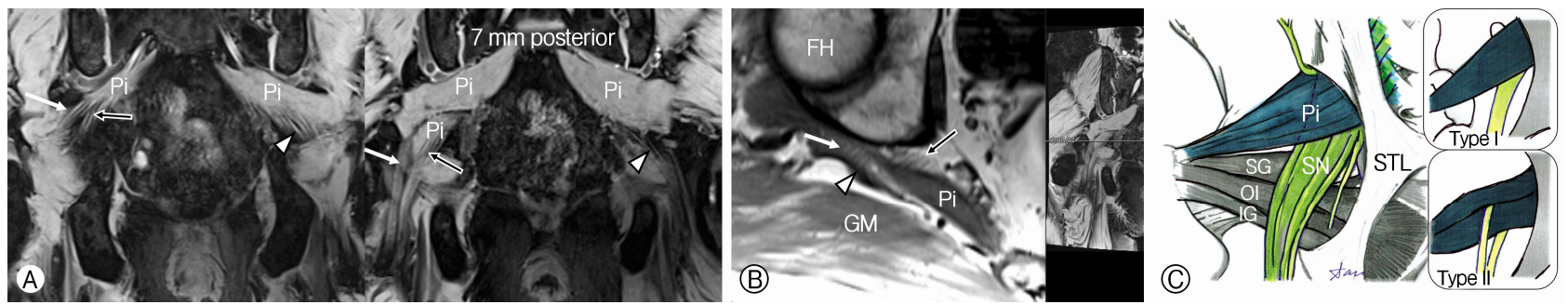

Fig. 2. Pelvic magnetic resonance imaging of failed back surgery syndrome associated with sciatic nerve (SN) entrapment. (A) Coronal T2weighted images revealing type II variation between the right SN and the piriformis. The left image shows the high division of the right SN. The peroneal division (white arrow) is located lateral to the tibial division (black arrow). The left SN (white arrow head) has a normal course running under the piriformis muscle in the form of type I variation. In the image on the right, the peroneal division (white arrow) couses under the main belly of the piriformis muscle. (B) Axial T1-weighted image showing the entrapment of the right $\mathrm{SN}$. The peroneal division (white arrow) is located behind the piriformis muscle and the tibial division (black arrow) is located in front of the piriformis muscle. Note the location of the inferior gluteal artery (white arrow head) immediately behind the peroneal division. (C) Anatomical variations between the SN and the piriformis muscle. Type I and II variations are shown on the right. FH: the head of the femur; GM: gluteus maximus muscle; STL: sacrotuberous ligament.
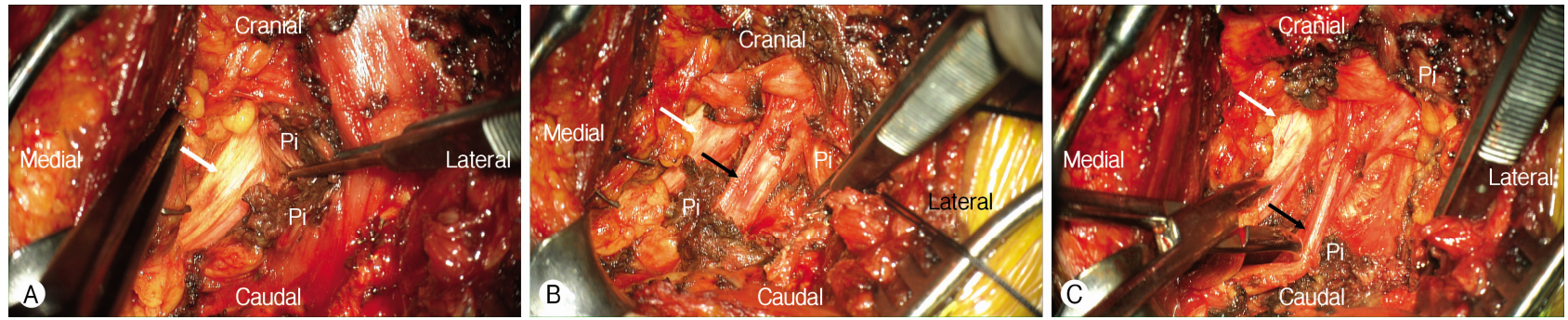

Fig. 3. Intraoperative images during decompression of the right sciatic nerve. (A) Initial exposure of the sciatic nerve (white arrow) with partial resection of the piriformis muscle (Pi). This nerve was found to be the tibial division via direct electrical stimulation. (B) An intraoperative image showing the tibial (white arrow) and peroneal divisions (black arrow) exposed by resecting the upper and lower bellies of the Pi. (C) An intraoperative image showing complete decompression of the tibial (white arrow) and peroneal divisions (black arrow) due to complete resection of the upper and lower bellys of the $\mathrm{Pi}$. 
The 4rth and 5th lumbar nerve roots combine with the first and second sacral nerve roots to form the common peroneal and tibial nerves, which together constitute the sciatic nerve. Six anatomical relationships between the sciatic nerve and piriformis muscle (Fig. 2C) were first described by Beaton and Anson $^{2)}$ in 1937. The conventional and most common relationship, type I, entails an undivided sciatic nerve that courses below the piriformis muscle. The type I sciatic nerve anatomy is prevalent in approximately $80 \%$ to $90 \%$ based on a cadaver study ${ }^{18)}$. The type II sciatic nerve is the most common variant, with a prevalence of $10 \%$ to $15 \%$, in which one division of the sciatic nerve passes through and the other below the piriformis muscle (Fig. 2C). Type III sciatic nerve is the second most common variant, with a prevalence of $1 \%$ to $3 \%$, in which one division of the sciatic nerve passes above and the other below the piriformis muscle. The remaining variants, type IV-VI, are quite rare, each with less than $1 \%$ prevalence ${ }^{9,15)}$. A recent study investigating the sciatic nerve variation based on 755 routine MRIs of the hip showed that the incidence of type II variation was $13 \%{ }^{23)}$. Most variants were associated with a split sciatic nerve at the level of the ischial tuberosity (73 out of $111,65.8 \%)^{23)}$.

The diagnosis of sciatic nerve entrapment may be strongly suggested by the patient's medical history, especially blunt trauma to the gluteal area, such as a fall ${ }^{3,20}$. Often, the cause is an exacerbated activity of hip rotators or prolonged sitting on hard surfaces ${ }^{20)}$. However, its onset is sometimes spontaneous. The typical patient complaints include buttock pain with or without ipsilateral radiation to the ipsilateral posterior thigh that sometimes extends below the knee to the calf, resembling typical sciatica ${ }^{20)}$. Buttock pain or sciatica may be exacerbated by activity of the lower extremities, such as hip adduction and internal rotation that stretches the pyriformis muscle $^{20)}$. However, the cardinal feature is sitting intolerance $^{3,20)}$. Numbness in various sensory distributions on the involved side are common, but motor weakness in a corresponding distribution is rare ${ }^{3)}$. The 2 most common consistent findings in the physical examination are tenderness to palpation of the greater sciatic notch and reproduction of the pain with maximum flexion, adduction, and internal rotation of the hip $^{3,5,20)}$.

EMG is important for diagnosis, but is not absolute, as we have seen in this case diagnosed as S1 radiculopathy. Findings of the pyriformis syndrome include denervation potentials of the hamstring muscles supplied by the peroneal division of the sciatic nerve, but the muscles innervated by the tibial division are usually spared ${ }^{10)}$. Additionally the inferior gluteal nerve that supplies the gluteus maximus may be compressed, and the muscle may suggest the sign of denervation ${ }^{10)}$. The paraspinal muscles innervated by the dorsal rami are spared. The MRI of the hip and pelvis is the most sensitive imaging study for evaluation of sciatic nerve anatomy ${ }^{6,9,17,23)}$. Variant relationship between the sciatic nerve and the piriformis muscle is routinely detected in MRI and is an important finding in patients with extraspinal sciatica ${ }^{23}$. Evaluation of a split sciatic nerve at the level of the ischial tuberosity in the MRI is an important diagnostic approach because the variant anatomy is rarely seen when the common peroneal and tibial components of the sciatic nerve are tightly opposed or appear as a single nerve at the level of the ischial tuberosity ${ }^{23}$.

\section{Causes of Failed Back Surgery Syndrome}

With the aging of the population, the frequency of degenerative lumbar disease is rapidly increasing, and accordingly, surgery for lumbar spinal stenosis has also increased. With the increasing rate of spine surgery, the number of patients with FBSS has increased. FBSS is a term embracing a constellation of conditions that describes persistent or recurring low back pain, with or without sciatica, following one or more spinal surgeries ${ }^{19)}$. A more functional definition of FBSS is based on results of lumbar spine surgery, which do not meet the pre-surgical expectations of the patient and surgeon ${ }^{24)}$. The factors contributing to FBSS may occur in the preoperative, intraoperative, and postoperative periods ${ }^{5)}$. FBSS following lumbar spine surgery for sciatica due to sciatic nerve entrapment can be attributed to inappropriate preoperative selection for surgery ${ }^{5)}$.

Differential diagnosis of sciatic nerve entrapment from lumbar nerve root compression may be difficult in some instances. Indeed, 2 out of 14 patients reported by Benson and Schutzer ${ }^{3}$ ) in 1999 had undergone spinal fusion for chronic disabling sciatica due to an incorrect diagnosis of spinal stenosis instead of sciatic nerve entrapment. The most characteristic symptoms of degenerative spinal stenosis are back pain associated with sciatica and neurogenic intermittent claudication $(\mathrm{NIC})^{21)}$. Patients with NIC usually present with bilateral leg complaints. However, sciatic nerve entrapment is mostly unilateral. The distribution of pain in the lower extremities is dependent on the area of stenosis ${ }^{1)}$. Unlikely the buttock pain associated with sitting intolerance in sciatic nerve entrapment, pain associated with spinal stenosis improved with sitting, trunk flexion, stooping or lying and aggravated with prolonged standing or lumbar extension. However, sitting or lying down is less effective in relieving the pain associated with advanced lumbar stenosis $^{1)}$. In severe cases, pain at rest or a neurogenic bladder can develop. In patients with central stenosis, the symptoms usually involve both the buttocks and the posterior thigh in a non-dermatomal distribution. However, in patients with lateral recess stenosis, symptoms are usually dermatomal because specific nerves are compressed ${ }^{14)}$. They experience higher pain during rest and at night and may show greater walking intolerance than those with central stenosis. The neurological examination is usually normal and with no limitation in straight leg raising and femoral stretch test ${ }^{14)}$. Thus, in cases of advanced central stenosis involving the lumbar spine, the diagnosis of 
sciatic nerve entrapment based on a brief interview is difficult. Careful evaluation of the patient's complaints along with the past history of buttock trauma and sitting intolerance is an important initial step in the diagnosis of this syndrome.

\section{Management of Sciatic Nerve Entrapment}

First-line therapy for sciatic nerve entrapment and deep gluteal syndrome entails blocks and physiotherapy, with or without botulinum toxin injection ${ }^{11)}$. Initial response to injection treatment or botox injection to the piriformis muscle is generally high $(70-80 \% \text { response rate })^{6,7,11)}$. However, conservative management ultimately fails in many patients with deep gluteal syndrome ${ }^{7,8,15)}$. Patients with sciatic nerve entrapment who do not respond to conservative treatment generally require surgical decompression. Traditionally, surgical treatment using a transgluteal approach has been used ${ }^{6,20,22)}$, but endoscopic decompression has recently become more common among orthopedic surgeons for deep gluteal syndrome ${ }^{11,15)}$.

However, we believe that the transgluteal approach using microscopic vision is a better option for complete dissection of the sciatic nerve pathway in the subgluteal space. In particular, intraoperative nerve stimulation facilitated localization and verification of tibial and peroneal divisions, which are major divisions of the sciatic nerve during dissection ${ }^{12}$. Plantar flexion of the foot was observed with low-intensity stimulation (1-2 mA) of the tibial division, and dorsiflexion was observed for stimulation in the peroneal division. In addition, stimulation of the inferior gluteal and posterior femoral cutaneous nerves caused contraction of the gluteus maximus muscle without eliciting foot movemen. The nerve supplying the piriformis muscle could be distinguished via isolated contraction of the piriformis muscle. In case 4 of type II variation, it is important to completely remove the lower belly of the piriformis, which is sandwiched between the tibial and peroneal divisions of the sciatic nerve (Fig. 3B), and complete decompression along the path of the sciatic nerve from the sciatic notch above to the ischial tuberosity below. A high division of the sciatic nerve, as in the current case (Fig. 3C) requires excision of the major belly of the piriformis muscle by partially resecting the sacrotuberous ligament to expose the proximal course of the sciatic nerve. The release of the pyriformis muscle has a minor effect on the power of external rotators and abductors of the hip joint $^{22)}$.

\section{CONCLUSION}

A lack of awareness and understanding of buttock pain with sitting intolerance, which is a characteristic symptom of sciatic nerve entrapment in the subgluteal space, may lead to needless lumbar spinal surgery which inevitably triggers FBSS.

\section{CONFLICTS OF INTEREST}

No potential conflict of interest relevant to this article was reported.

\section{REFERENCES}

1. Amundsen T, Weber H, Nordal HJ, Magnaes B, Abdelnoor M, Lilleâs F: Lumbar spinal stenosis: Conservative or surgical management?: A prospective 10-year study. Spine (Phila Pa 1976) 25:1424-1435, 2000

2. Beaton LE, Anson BJ: The relationship of the sciatic nerve and its subdivisions to the piriformis muscle. Anat Rec 70:1-5, 1938

3. Benson ER, Schutzer SF: Posttraumatic piriformis syndrome: diagnosis and results of operative treatment. J Bone Joint Surg Am 81:941-949, 1999

4. Cass SP: Piriformis syndrome: A cause of nondiscogenic sciatica. Curr Sports Med Rep 14:41-44, 2015

5. Chan CW, Peng P: Failed back surgery syndrome. Pain Med 12:577-606, 2011

6. Filler AG, Haynes J, Jordan SE, Prager J, Villablanca JP, Farahani $\mathrm{K}$, et al.: Sciatica of nondisc origin and piriformis syndrome: diagnosis by magnetic resonance neurography and interventional magnetic resonance imaging with outcome study of resulting treatment. J Neurosurg Spine 2:99-115, 2005

7. Fishman LM, Anderson C, Rosner B: BOTOX and physical therapy in the treatment of piriformis syndrome. Am J Phys Med Rehabil 81:936-942, 2002

8. Fishman LM, Dombi GW, Michaelsen C, Ringel S, Rozbruch J, Rosner B, et al.: Piriformis syndrome: Diagnosis, treatment, and outcome-a 10-year study. Arch Phys Med Rehabil 83:295301, 2002

9. Hernando MF, Cerezal L, Pérez-Carro L, Abascal F, Canga A: Deep gluteal syndrome: anatomy, imaging, and management of sciatic nerve entrapments in the subgluteal space. Skeletal Radiol 44:919-934, 2015

10. Hughes SS, Goldstein MN, Hicks DG, Pellegrini VD, Jr.: Extrapelvic compression of the sciatic nerve. An unusual cause of pain about the hip: report of five cases. J Bone Joint Surg Am 74:1553-1559, 1992

11. Kay J, de Sa D, Morrison L, Fejtek E, Simunovic N, Martin $\mathrm{HD}$, et al.: Surgical management of deep gluteal syndrome causing sciatic nerve entrapment: A systematic review. Arthroscopy 33:2263-2278.e2261, 2017

12. Kim D, Choi J, Son B: Identification of safe zone with intraoperative neurophysiological monitoring during surgical removal of peripheral nerve tumor. Nerve 2:78-80, 2016

13. Konstantinou K, Dunn KM: Sciatica: review of epidemiological studies and prevalence estimates. Spine (Phila Pa 1976) 33:24642472, 2008

14. Lee SY, Kim TH, Oh JK, Lee SJ, Park MS: Lumbar stenosis: A recent update by review of literature. Asian Spine J 9:818-828, 2015

15. Martin HD, Shears SA, Johnson JC, Smathers AM, Palmer IJ: The endoscopic treatment of sciatic nerve entrapment/deep gluteal syndrome. Arthroscopy 27:172-181, 2011 
16. Mixter WJ, Barr JS: Rupture of the intervertebral disc with involvement of the spinal canal. N Engl J Med 211:210-215, 1934

17. Moore KR, Tsuruda JS, Dailey AT: The value of MR neurography for evaluating extraspinal neuropathic leg pain: a pictorial essay. AJNR Am J Neuroradiol 22:786-794, 2001

18. Natsis K, Totlis T, Konstantinidis GA, Paraskevas G, Piagkou $\mathrm{M}$, Koebke J: Anatomical variations between the sciatic nerve and the piriformis muscle: A contribution to surgical anatomy in piriformis syndrome. Surg Radiol Anat 36:273-280, 2014

19. North RB, Campbell JN, James CS, Conover-Walker MK, Wang $\mathrm{H}$, Piantadosi S, et al.: Failed back surgery syndrome: 5-year follow-up in 102 patients undergoing repeated operation. Neurosurgery 28:685-690, 1991

20. Papadopoulos EC, Khan SN: Piriformis syndrome and low back pain: A new classification and review of the literature. Orthop Clin North Am 35:65-71, 2004

21. Rauschning W: Normal and pathologic anatomy of the lumbar root canals. Spine (Phila Pa 1976) 12:1008-1019, 1987

22. Robinson DR: Piriformis syndrome in relation to sciatic pain. Am J Surg 73:355-358, 1947

23. Varenika V, Lutz AM, Beaulieu CF, Bucknor MD: Detection and prevalence of variant sciatic nerve anatomy in relation to the piriformis muscle on MRI. Skeletal Radiol 46:751-757, 2017

24. Waguespack A, Schofferman J, Slosar P, Reynolds J: Etiology of long-term failures of lumbar spine surgery. Pain Med 3:1822, 2002

25. Yeoman W: The relation of arthritis of the sacro-iliac joint to sciatica, with an analysis of 100 cases. Lancet 212:1119-1123, 1928 